Cinémas

Revue d'études cinématographiques

Journal of Film Studies

\title{
Stéphane-Albert Boulais (dir.), Le cinéma au Québec. Tradition et modernité, Montréal, Fides, 2006, 349 p.
}

\section{Karine Bertrand}

Volume 19, numéro 1, automne 2008

Les transformations du cinéma

URI : https://id.erudit.org/iderudit/029504ar

DOI : https://doi.org/10.7202/029504ar

Aller au sommaire du numéro

Éditeur(s)

Cinémas

ISSN

1181-6945 (imprimé)

1705-6500 (numérique)

Découvrir la revue

Citer ce compte rendu

Bertrand, K. (2008). Compte rendu de [Stéphane-Albert Boulais (dir.), Le cinéma au Québec. Tradition et modernité, Montréal, Fides, 2006, 349 p.] Cinémas, 19(1), 183-189. https://doi.org/10.7202/029504ar d'utilisation que vous pouvez consulter en ligne.

https://apropos.erudit.org/fr/usagers/politique-dutilisation/ 
Stéphane-Albert Boulais (dir.), Le cinéma au Québec. Tradition et modernité, Montréal, Fides, 2006, 349 p.

Le cinéma québécois connaît présentement une vague de sympathie peu commune. Les films attirent le public dans les salles et les artisans se font de plus en plus nombreux. De nouveaux auteurs émergent qui répondent aux sensibilités actuelles pour nommer et représenter les problématiques de la société. On a même parlé de "nouvelle vague» pour caractériser le travail d'un groupe de jeunes de la relève qui, de leur aveu même, tirent leur inspiration de l'héritage de l'éthique et de l'esthétique du cinéma direct pour créer des œuvres à la fois personnelles et susceptibles d'attirer un public fidèle.

Cette ascendance d'une époque fondatrice dans laquelle baigne le cinéma au Québec et pour laquelle les pionniers (Proulx, Jutra, Perrault, etc.) semblent occuper une place centrale est parfaitement ressentie dans l'ouvrage dirigé par le professeur de cinéma et écrivain Stéphane-Albert Boulais, Le cinéma au Québec. Tradition et modernité. Le collectif réunit plusieurs spécialistes du cinéma, dont la plupart ont à leur actif plus d'une trentaine d'années d'expérience dans le domaine. Les thèmes qui en émanent sont d'abord ceux de la mémoire et du pays, parfums de terre et de lumière qui renvoient à l'époque du bonimenteur et de la magie première qui accompagne la naissance du $7^{\mathrm{e}}$ art, et qui se transforme avec l'arrivée de nouvelles techniques en une effervescence qui atteint son apogée avec l'émergence du direct dans les années soixante. C'est d'ailleurs cette période comprise entre 1960 et 1980 qui sera re-visitée par la majorité des auteurs, lesquels s'appliquent à rendre justice (pour ne pas dire rendre hommage) aux ouvres issues de cette époque et qui continuent d'influencer les jeunes générations de cinéastes comme Francis Leclerc, Denis Villeneuve, Denis Chouinard ou Philippe Falardeau. 
Viendront se greffer à ces derniers thèmes quelques textes qui nous font pénétrer dans une ère plus contemporaine, en traitant par exemple de l'oralité au cinéma, de l'ère du numérique, de la rencontre des cultures et en esquissant finalement quelques portraits de cinéastes comme Denys Arcand et Pierre Perrault, dont les œuvres ont profondément marqué la cinématographie québécoise.

D’entrée de jeu, le texte de Pierre Véronneau «Écrire en dépit des trous de mémoire", nous invite à prendre connaissance des premiers balbutiements de l'industrie du cinéma au Québec. Citant comme source principale le groupe de recherche GRAFICS, sur le cinéma des premiers temps, l'auteur nous informe de cette époque charnière (et méconnue) située entre le muet et le parlant, où le cinéma québécois a peu à peu forgé son identité, à travers l'expression d'une oralité aux couleurs locales qui a favorisé l'appropriation culturelle et le développement d'un nouveau langage. Ce faisant, nous apprenons cette volonté inébranlable des cinéastes québécois qui, cent ans plus tôt, manifestaient ce même désir de pouvoir créer et diffuser un produit intègre, éloigné des représentations étrangères et de leur contenu uniforme. Dans la même veine, Esther Pelletier et Steven Morin (avec la collaboration de Karine Latulippe) retracent l'histoire du scénario au Québec de 1913 à 2003, en soulignant les principales transformations de l'écriture scénaristique en fonction des exigences de l'époque et bien entendu, de l'industrie. Le film documentaire occupant presque entièrement le paysage cinématographique québécois de 1920 à 1970, on nous apprend que le scénario ne connaîtra son heure de gloire qu'avec le déclin (relatif) du cinéma documentaire et le développement d'une industrie qui encouragera désormais le cinéma de fiction. S'ensuit une description fort détaillée de l'émergence du scénario à partir de la moitié de la décennie 70 et des principaux scénaristes qui ont marqué cette période. Or, si l'ensemble du texte s'avère généreux en matière d'informations historiques, on peut déplorer le fait que peu d'espace soit alloué à dresser un portrait du scénario au présent, ou même à présager une direction vers laquelle l'écriture scénaristique se dirige. Les auteurs se contentent de mentionner l'hétérogénéité et la mixité du 
scénario moderne, à présent devenu "une étape incontournable pour permettre à un film ou à un produit télévisuel de voir le jour»(p. 45). Philippe Lemieux poursuit quant à lui ce survol historique du cinéma québécois en retraçant cette fois son évolution technologique, depuis ses déboires avec l'Église au début du $\mathrm{XX}^{\mathrm{e}}$ siècle jusqu'à l'arrivée et l'exploitation du numérique dans les années quatre-vingt. Outre un rappel important de la contribution québécoise au développement et à l'évolution du cinéma numérique à l'échelle occidentale, l'intérêt de ce texte se situe au niveau de la réflexion qu'il suscite par rapport au débat qui oppose les puristes (ou traditionalistes) aux défenseurs du numérique. Alors que les premiers anticipent par le biais du numérique une trop grande fissure avec le réel et la disparition d'une esthétique propre au genre documentaire, l'auteur affirme que «le tournage en vidéo numérique adopte une texture et une esthétique qui contribue à créer l'illusion documentaire", en plus de permettre au cinéaste de travailler efficacement avec un budget restreint, une réalité incontournable ici au Québec (p. 73). La démocratisation du cinéma québécois passerait donc par le développement d'une technologie abordable, facilement accessible et qui serait bénéfique pour tous les genres, du cinéma d'auteur au documentaire, sans oublier le cinéma d'animation qui occupe une place de choix au Québec.

Pour conclure la partie plus historique de ce parcours, le texte de Germain Lacasse, "L'accent aigu du cinéma oral ", s'applique à démontrer, de façon fort convaincante d'ailleurs, la présence d'une oralité qui demeure à ce jour une partie intrinsèque de notre cinéma. L'auteur retrace d'abord les origines de cette oralité à l'époque du bonimenteur (1900-1930), qui agissait à la fois à titre de conférencier et surtout de médiateur entre le public et l'écran. Ensuite il nous explique le rôle fondamental que celui-ci a joué dans l'élaboration d'une pratique antihégémonique du cinéma, en invitant les spectateurs à participer à la construction de sens d'une ouvre qu'il adaptait au contexte local. Qui plus est, Lacasse nous révèle la continuité de cette tradition orale à travers le temps, d'abord par l'intermédiaire des prêtres-cinéastes qui se promenaient de village en village pour présenter leurs documentaires, souvent accompagnés d'un 
commentaire prononcé en direct devant leur public. Par la suite, l'arrivée du cinéma direct et d'une technique (caméra mobile, son et image synchrones) qui permet au cinéaste comme au spectateur de se retrouver au cœur de l'action, contribue à perpétuer ce dialogue incessant entre le public et le film. En suggérant ce lien intime qui existe entre la parole et l'image, en soulignant cette invitation tacite de la caméra à un rassemblement communautaire, l'auteur nous rappelle l'importance de faire entendre cette voix (omnisciente ou non) qui porte un discours intérieur mais aussi celui de toute une nation, pour qui l'oralité a permis de survivre (culturellement) à l'invasion d'une industrie étrangère.

Toujours sur le thème de l'oralité, le texte de Marie-Chantal Killeen, "Un narrateur sans organe. Une problématisation de la voix off dans Léolo", que l'on découvre dans la seconde partie de l'ouvrage, présente une parfaite continuité avec celui de Lacasse, en proposant d'examiner le rôle de la voix off dans le célèbre film de Lauzon et de répondre à la question «qui ou qu'est-ce qui parle dans Léolo?» (p. 125). Utilisant comme base théorique le concept de la voix acousmatique développé dans l'ouvrage $L a$ voix au cinéma de Michel Chion (1984) l'auteure nous soumet un texte original, fort bien écrit, qui ajoute à notre appréciation $\mathrm{du}$ film et à sa transmutation en poème pur, en détachant la voix du corps qu'elle est censée représenter pour l'identifier à un «narrateur sans organe» (p. 138). À travers l'analyse de Killeen, qui attribue à cette voix off une fonction d'identification et un rôle de complice, nous prenons soudainement conscience de la présence de cette voix fantomatique, flottante qui ne peut logiquement appartenir au personnage (rappelons-nous son état catatonique à la toute fin du film) et qui confirme par ailleurs son influence sur le climat troublant présent tout au long $\mathrm{du}$ film. En somme un brillant hommage à un long métrage qui ne laisse personne indifférent et qui témoigne de la versatilité du cinéma québécois.

Dans un tout autre registre, le thème de la mémoire est abordé différemment par quelques-uns des collaborateurs de cet ouvrage, qui choisissent de poser un regard plutôt nostalgique que critique sur une époque révolue de cette cinématographie. 
Alors qu'Yves Lever s'attaque à la mémoire collective, au territoire et à l'identité, en essayant de départager ce qui doit rester (l'âge d'or du direct et tout ce qui vient avec) de ce qui doit être renouvelé (cette sempiternelle représentation des Québécois en victimes consentantes), les professeurs Jacques Michaud et Yves Alcaïdé posent un regard plus personnel sur leur parcours de cinéphiles. Le premier en reliant ses origines abitibiennes à différentes œuvres documentaires et de fiction (Proulx, Perrault, Devlin), et le second en exprimant le point de vue de l'étranger qui découvre peu à peu le cinéma de son pays d'accueil. Deux témoignages qui, libérés des contraintes d'une analyse théorique rigide, permettent d'apprécier les qualités inhérentes à chacune des œuvres mentionnées par les auteurs, comme quoi nous devons parfois laisser de côté nos prétentions académiques, nous re-positionner en tant que simples admirateurs du cinéma.

Divers textes dispersés dans l'ouvrage participent d'ailleurs à cet exercice de découverte et de contemplation, en nous faisant connaître sous un jour nouveau des cinéastes ayant contribué de façon significative à l'édification d'une cinématographie riche et variée. D'abord, on ne peut passer à côté de l'éloge dithyrambique de Pierre Pageau à l'égard du cinéaste anglophone Colin Low, initiateur du programme onéfien "Challenge for Change». En plus de nous informer sur le rôle important qu'a joué l'ONF dans cette rencontre culturelle entre le Québec et le reste du pays, l'auteur fait étalage des similarités qui existent entre le cinéma anglophone et francophone, entre autres en faisant un parallèle entre l'œuvre de Perrault et celle de Colin Low. Ce faisant, nous apprenons que la question identitaire dépasse les frontières du Québec pour s'étendre au cinéma canadien qui tente tant bien que mal de se démarquer face à la machine industrielle hollywoodienne. Autre portrait intéressant, celui de Jean-François Caron sur le metteur en scène et cinéaste Robert Lepage, et dans lequel les thèmes de la mémoire, de l'écriture cinématographique et de l'avenir technologique du cinéma au Québec rappellent le ton général de l'ouvrage.

Enfin, pour compléter cette mosaïque du cinéma au Québec, on ne peut passer à côté du texte de Denis Bachand et Annie Lise Clément, "La rencontre des cultures dans le cinéma 
québécois : violence et altérité », un texte indispensable parce qu'il est à peu près le seul à élargir le spectre des thèmes exploités pour y inclure dans sa définition du cinéma québécois celui de l'étranger, de l'immigrant, et de rendre compte de manière réaliste de l'impact de cette rencontre sur les différentes cultures au Québec. Le texte résume admirablement bien le paradigme identitaire de notre époque, en soulignant les difficultés d'intégration et la présence dans la société québécoise d'une forme de crispation identitaire, qui bien souvent conduit à la violence et à l'exclusion de l'étranger. Qui plus est, ils constatent l'absence, jusqu'au milieu des années quatre-vingt, d'une reconnaissance du cinéma réalisé par des immigrants québécois. Cela nous amène à nous interroger sur la définition attribuée au mot Québécois, qui semble placer le film autochtone ou immigrant dans une catégorie à part. À cet égard, on peut reprocher à l'ouvrage l'absence de textes sur les ouvres créées par des cinéastes autochtones et dont plusieurs sont des documentaires traitant de la dure réalité amérindienne et des relations conflictuelles entre les Blancs et les membres des Premières Nations du Québec. Tout en appréciant l'unicité et la richesse des œuvres qui découlent de la période du direct, on peut tout de même se demander s'il faut être un Québécois de souche, "pure laine» comme on dit, ou avoir fréquenté Perrault et sa bande pour obtenir une place de choix au sein de notre cinéma. Peut-être le temps est-il venu de redéfinir cette notion de québécité chère à une époque maintenant révolue, pour y inclure les minorités (amérindiens et immigrants) qui participent activement à la construction d'un cinéma de plus en plus diversifié. Dans la même veine, Marion Froger, dans le texte "Le cinéma québécois à l'épreuve de la communauté", oppose le cinéma institutionnel au cinéma communautaire et examine le rôle du documentaire engagé en faisant état de la fonction parfois thérapeutique de celui-ci. La description du documentaire bouleversant de Maurice Bulbulian, Chroniques de Nitinaht (1997) présente l'exemple parfait d'une œuvre produite en collaboration étroite avec la communauté, laquelle dictait le rythme du film en fonction du processus de guérison qu'elle vivait, le médium fournissant quant à lui l'espace virtuel où les liens se 
sont peu à peu ressoudés. Bref, un texte qui souligne l'importance d'atteindre et de conserver son identité à travers cet équilibre instable entre "le rapport à soi et l'ouverture aux autres» (p. 220).

En somme, l'ouvrage nous amène à nous interroger sur la condition du cinéma au Québec, en suggérant une hésitation du peuple québécois à plonger ses racines dans le présent et oser regarder en avant pour mieux rêver l'avenir. Ce cinéma s'est-il laissé séduire par des rêves de grandeur jusqu'à en être réduit à devenir un produit du grand marché culturel? Plus important encore, arrive-t-il toujours à nous éblouir? Le texte de StéphaneAlbert Boulais «Le langage de l'inexprimable» répond partiellement à ces questions en évoquant le rôle du cinéma comme porteur de liberté et en nous apprenant que nous pouvons à nouveau être séduits par un film comme Le Marais (Nguyen, 2002) et assister à l'éclosion d'une nouvelle génération de cinéastes connus sous l'appellation de "Kino", "qui font revivre, à leur façon, la belle folie de l'inexprimable auquel l'écriture cinéma au Québec doit beaucoup de sa vitalité»(p. 318). Quoi qu'il en soit, si le cinéma est d'abord une écriture, comme le proclame le poète en guise d'introduction de l'ouvrage, on apprend à la lecture des différents chapitres qu'il est peut-être davantage une oralité, un langage, ce langage de l'inexprimable qui hésite, balbutie, trébuche, se relève (p. 301). Un langage qui tente de se donner un miroir adéquat, en contribuant au façonnement d'une image où le rêve et le réel se confondent pour traduire une réalité mouvante.

Karine Bertrand

Université d'Ottawa

RÉFÉRENCES BIBLIOGRAPHIQUES

Chion 1984 : Michel Chion, La voix au cinéma, Paris, Cahiers du cinéma, 1984. 